Leukemia (2019) 33:2116-2120

https://doi.org/10.1038/s41375-019-0443-7

Chronic myelogenous leukemia

\title{
Skeletal muscle toxicity associated with tyrosine kinase inhibitor therapy in patients with chronic myeloid leukemia
}

\author{
L. Janssen ${ }^{1,2}$ - S. J. C. M. Frambach ${ }^{3,4}$ - N. A. E. Allard ${ }^{1}$ - M. T. E. Hopman ${ }^{1}$ - T. J. J. Schirris ${ }^{3,4}$ - N. C. Voermans ${ }^{5}$. \\ R. J. Rodenburg ${ }^{4,6}$ - N. M. A. Blijlevens ${ }^{2}$ - S. Timmers ${ }^{1,7}$
}

Received: 3 December 2018 / Revised: 21 February 2019 / Accepted: 22 February 2019 / Published online: 14 March 2019

(c) The Author(s) 2019. This article is published with open access

\section{To the Editor:}

Up to $80 \%$ of CML patients using tyrosine kinase inhibitors (TKIs) reports muscle complaints [1]. These muscle complaints are strongly related to the presence of fatigue and contributes to both diminished disease control [2] and impaired quality of life [3]. Although the mechanism by which TKIs cause muscle complaints is poorly understood, mitochondrial dysfunction has been suggested to play a pivotal role in TKI-induced cardiac muscle toxicity [4, 5]. We assessed whether TKIs disturb skeletal muscle mitochondrial density and function (cellular level), and if this translates into alterations in muscle contractile function (muscle tissue level) and maximal exercise performance (whole-body level). To gain a better insight into TKI-induced muscle complaints, these outcomes were compared between CML patients with and without muscle complaints. Written informed consent was obtained from the participants prior to study enrollment. This study was approved by the Local Committee on Research Involving Human Subjects of the region Arnhem and Nijmegen,

These authors contributed equally: S. J. C. M. Frambach, N. A. E. Allard and N. M. A. Blijlevens, S. Timmers

$\triangle$ N. M. A. Blijlevens

Nicole.Blijlevens@radboudumc.nl

$\triangle$ S. Timmers

Silvie.Timmers@radboudumc.nl

1 Radboud Institute for Health Sciences, Department of Physiology, Radboud University Medical Center, Nijmegen, The Netherlands

2 Radboud Institute for Health Sciences, Department of Hematology, Radboud University Medical Center, Nijmegen, The Netherlands

3 Department of Pharmacology and Toxicology, Radboud Institute for Molecular Life Sciences, Nijmegen, The Netherlands the Netherlands and registered at The Netherlands Trial Registry (NTR6373).

A total of twenty $\mathrm{Ph}^{+}$CP-CML patients on TKI therapy aged $\geq 18$ were recruited from the Department of Hematology at the Radboud University Medical Center (Nijmegen, The Netherlands). CML patients were assigned to a group with $(\mathrm{CML}+\mathrm{MC}, N=10)$ or without (CML - MC, $N=10)$ muscle complaints (MC) on the basis of presence, onset and course of muscle cramps, pain, and/or weakness. This was quantified on a Likert scale from 1 (not at all) to 4 (very much) resulting in significant different scores between $\mathrm{CML}+\mathrm{MC}$ and $\mathrm{CML}-\mathrm{MC}$ (median 4.0 (interquartile range (IQR) 3.0-4.0) and 2.5 (IQR 2.0-3.0), respectively; $P=0.002$ ). The Brief Fatigue Inventory (BFI) [6] was used to compare the degree of fatigue in CML patients, showing higher fatigue levels in $\mathrm{CML}+\mathrm{MC}$ when compared to CML - MC $(3.58 \pm 2.19$ and $0.95 \pm 1.11$, respectively; $P=$ 0.005). Ten control participants were matched on group level for age, gender, BMI and physical activity level, assessed by The Short Questionnaire to Assess HealthEnhancing Physical Activity (SQUASH) [7]. Subjects were ineligible if they had hereditary muscle defects, diabetes mellitus, hypo- or hyperthyroidism, severe electrolyte disturbances, or used co-medication known to cause muscle symptoms or have an effect on mitochondrial function (e.g. statins, steroids, and metformin). Furthermore, subjects

4 Center for Systems Biology and Bioenergetics, Radboud Center for Mitochondrial Medicine, Radboud University Medical Center, Nijmegen, The Netherlands

5 Department of Neurology, Donders Institute for Brain, Cognition, and Behaviour, Radboud University Medical Center, Nijmegen, the Netherlands

6 Radboud Center for Mitochondrial Medicine, Translational Metabolic Laboratory, Department of Pediatrics, Radboud University Medical Center, Nijmegen, The Netherlands

7 Human and Animal Physiology, Wageningen University, Wageningen, The Netherlands 
with contra-indications for maximal exercise testing according to the ACC/AHA guidelines [8] and muscle biopsy (e.g. anticoagulant therapy, bleeding disorders) were excluded. The demographic and hematological characteristics shown in Table 1 are not statistically significant different between CML patients and controls and between CML + MC and CML - MC, except for a higher Charlson Comorbidity Index [9] score in CML patients when compared to controls (median 2.0 (IQR 2.0-2.0) and 0.0 (IQR $0.0-0.0$ ), respectively; $P<0.001)$ caused by the presence of CML. All participants completed the study protocol, i.e. a vastus lateralis muscle biopsy, electrical quadriceps femoris stimulations, and an incremental cycling test.

Vastus lateralis muscle needle biopsies were performed under local anesthesia in overnight fasted state and processed for mitochondrial measurements according to standard lab techniques as previously published [10]. Citrate synthase activity, a marker for mitochondrial density, was not different between CML patients and controls $(195 \pm 80 \mathrm{mU} / \mathrm{mg}$ protein and $171 \pm 30 \mathrm{mU} / \mathrm{mg}$ protein, respectively, $P=0.24$ ) and between $\mathrm{CML}+\mathrm{MC}$ and $\mathrm{CML}-\mathrm{MC} \quad(P=0.33)$.
Furthermore, mitochondrial function, assessed by ATP production capacity (Fig. 1a, c) and $\left[1-{ }^{14} \mathrm{C}\right]$-pyruvate oxidation rates in the presence of malate or carnitine (Fig. 1b, d) was not different between groups.

Maximal voluntary muscle strength of the dominant quadriceps femoris muscle [11], did neither differ between CML patients and controls $(8.3 \pm 2.0 \mathrm{~N} / \mathrm{kg}$ and $7.9 \pm 1.8 \mathrm{~N} / \mathrm{kg}$, respectively; $P=0.59)$, nor between $\mathrm{CML}+\mathrm{MC}$ and CML-MC ( $P=0.97)$. Resistance to fatigue was assessed by electrically stimulating the quadriceps femoris muscle repetitively at $40 \%$ of the MVC using $30 \mathrm{~Hz}$ bursts of onesecond duration every other second for two minutes [11]. This fatigue protocol resulted in a significantly larger force decline in CML patients as compared to controls $(31.8 \pm$ $8.7 \%$ and $23.6 \pm 7.7 \%$, respectively; $P=0.010$; Fig. 1e). Although a similar fatigability pattern was observed between CML + MC and CML - MC (force decline $29 \pm$ $9 \%$ and $34 \pm 9 \%$, respectively; $P=0.24$ ), the contractile properties of the quadriceps femoris muscle during repeated stimulation were explored in more detail. After two minutes of stimulation $\mathrm{CML}+\mathrm{MC}$ showed a

Table 1 Subject and hematological characteristics

\begin{tabular}{|c|c|c|c|c|c|c|}
\hline Characteristics & CML patients & Controls & $P$ value & $\mathrm{CML}+\mathrm{MC}$ & CML - MC & $P$ value \\
\hline Subject number, $N$ & 20 & 10 & - & 10 & 10 & - \\
\hline Gender, male/female & $14 / 6$ & $7 / 3$ & 1.00 & $7 / 3$ & $7 / 3$ & 1.00 \\
\hline Age, years & $54 \pm 8$ & $58 \pm 7$ & 0.25 & $55 \pm 9$ & $54 \pm 8$ & 0.86 \\
\hline BMI, $\mathrm{kg} / \mathrm{m}^{2}$ & $25.8 \pm 4.1$ & $27.5 \pm 5.4$ & 0.34 & $25.8 \pm 3.6$ & $25.8 \pm 4.8$ & 0.97 \\
\hline Physical activity, METmin/week; median (IQR) & $2288(1545-4982)$ & $2850(1965-4868)$ & 0.53 & $2541(1689-5488)$ & $2070(1403-4570)$ & 0.63 \\
\hline Smoker, \% & 0 & 0 & - & 0 & 0 & - \\
\hline Age at Dx, years & $46 \pm 8$ & N/A & - & $47 \pm 7$ & $46 \pm 9$ & 0.78 \\
\hline BCR-ABL level, $N(\%)$ & & N/A & - & & & 1.00 \\
\hline No MMR & $1(5)$ & & & $1(10)$ & $0(0)$ & \\
\hline MMR or deeper & $19(95 \%)$ & & & $9(90)$ & $10(100)$ & \\
\hline TKI, $N(\%)$ & & & - & & & 0.89 \\
\hline Imatinib & $10(50)$ & N/A & & $6(60)$ & $4(40)$ & \\
\hline Dasatinib & $5(25)$ & N/A & & $2(20)$ & $3(30)$ & \\
\hline Nilotinib & $2(10)$ & N/A & & $1(10)$ & $1(10)$ & \\
\hline Bosutinib & $2(10)$ & N/A & & $1(10)$ & $1(10)$ & \\
\hline Ponatinib & $1(5)$ & N/A & & $0(0)$ & $1(10)$ & \\
\hline Duration of current TKI therapy, months; median (IQR) & $42.5(21.0-114.8)$ & N/A & - & $66.5(19.0-121.5)$ & $38.0(16.5-73.3)$ & 0.63 \\
\hline Prior TKIs, $N$; median (IQR) & $0.5(0.0-1.0)$ & N/A & - & $0.0(0.0-1.0)$ & $1.0(0.0-1.0)$ & 0.53 \\
\hline Charslon Comorbidity Index; median (IQR) & $2.0(2.0-2.0)$ & $0.0(0.0-0.0)$ & $<0.001$ & $2.0(2.0-2.0)$ & $2.0(2.0-2.0)$ & 0.74 \\
\hline Potassium, mmol/l & $3.9 \pm 0.28$ & $4.0 \pm 0.20$ & 0.50 & $3.9 \pm 0.21$ & $4.0 \pm 0.34$ & 0.76 \\
\hline Magnesium, mmol/l & $0.82 \pm 0.06$ & $0.84 \pm 0.06$ & 0.49 & $0.82 \pm 0.05$ & $0.83 \pm 0.06$ & 0.63 \\
\hline Phosphate, mmol/l & $0.82 \pm 0.12$ & $0.91 \pm 0.17$ & 0.11 & $0.83 \pm 0.10$ & $0.81 \pm 0.15$ & 0.75 \\
\hline Calcium, mmol/1 & $2.29 \pm 0.09$ & $2.30 \pm 0.06$ & 0.68 & $2.29 \pm 0.09$ & $2.29 \pm 0.10$ & 0.91 \\
\hline Albumin, g/l & $38.7 \pm 2.5$ & $37.3 \pm 2.0$ & 0.13 & $39.1 \pm 2.8$ & $38.3 \pm 2.2$ & 0.48 \\
\hline TSH, mE/l; median (IQR) & $2.1(1.4-2.6)$ & $2.0(1.3-2.8)$ & 0.90 & $2.0(0.8-2.9)$ & $2.1(1.5-2.7)$ & 0.61 \\
\hline CK, U/l; median (IQR) & $137(89-236)$ & $120(109-166)$ & 0.61 & $169(103-312)$ & $127(84-216)$ & 0.90 \\
\hline
\end{tabular}

Values are presented as mean \pm SD unless indicated otherwise. There were no significant differences in subject and hematological characteristics between CML patients, except for a higher Charslon Comorbidity Index score in CML patients Also, there were no significant differences between CML patients with and without TKI induced muscle complaints

$M C$ muscle complaints, $B M I$ body mass index, $M E T$ metabolic equivalent of task, $I Q R$ interquartile range, $D x$ diagnosis, $M M R$ major molecular response, $T K I$ tyrosine kinase inhibitor, $T S H$ thyroid-stimulating hormone, $C K$ creatine kinase 

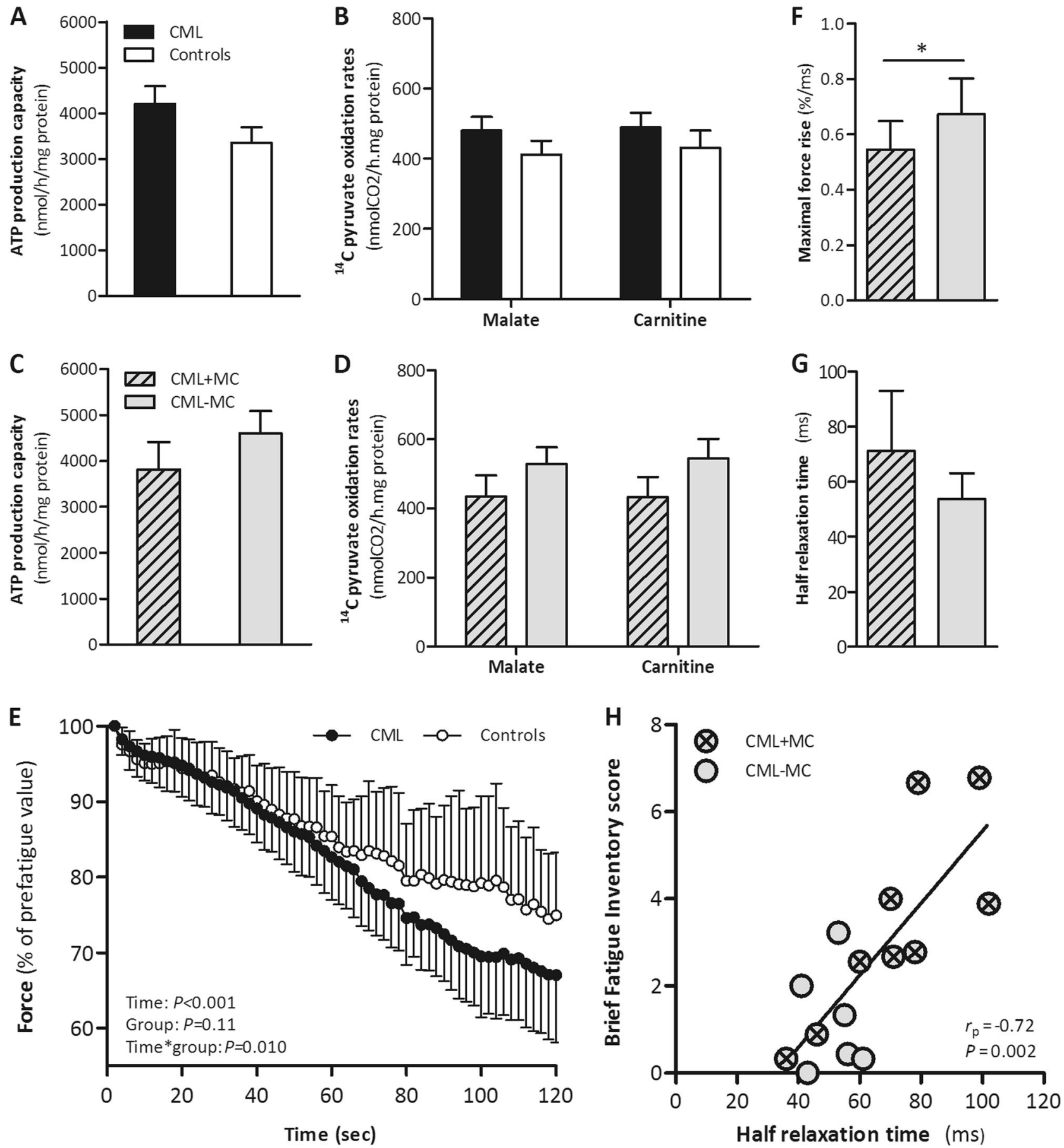

Fig. 1 Skeletal muscle mitochondrial function and muscle function parameters measured in CML patients and controls. a ATP production rate normalized to muscle protein content and (b) $\left[1-{ }^{14} \mathrm{C}\right]$-pyruvate oxidation rates in the presence of malate and carnitine normalized to muscle protein content measured in mitochondrial fractions from fresh vastus lateralis muscle biopsies were not affected by TKI use. There were also no differences in (c) ATP production rate and $(\mathbf{d})\left[1-{ }^{14} \mathrm{C}\right]$ pyruvate oxidation rates in the presence of malate and carnitine between CML + MC and CML-MC. e Force decline expressed as percentage of the prefatigue value during two minutes repetitive electrical stimulation of the quadriceps femoris muscle shows a higher level of muscle fatigue in CML patients compared to controls. Force

responses are plotted every second during the complete $(120 \mathrm{sec})$ fatigue protocol. f Maximal force rise after two minutes of electrical quadriceps femoris stimulation was significantly lower in CML + MC when compared to CML-MC. g Quadriceps femoris muscle in CML + MC showed a tendency toward longer relaxation time after two minute repetitive stimulation when compared to CML - MC. h Reported fatigue by CML patients (assessed by the Brief Fatigue Inventory) correlates with half relaxation time in quadriceps femoris muscle after two minutes repetitive electrical stimulation $(N=15)$. Data are presented as means \pm SEM for $\mathbf{a}-\mathbf{d}$; and means \pm SD for $\mathbf{e}-\mathbf{g}$. $* P$ value $<0.05$ is considered statistically significant 
significantly lower maximal force rise (maximal slope of force increment normalized for peak force) compared to CML-MC $(0.54 \pm 0.10 \% / \mathrm{ms}$ and $0.67 \pm 0.13 \% / \mathrm{ms}$, respectively; $P=0.038$; Fig. 1f) and a tendency toward longer half relaxation time (time taken for force to decline from 50 to $25 \%$ of the peak force; $P=0.07$; Fig. $1 \mathrm{~g}$ ). The half relaxation time at the end of the fatigue protocol strongly correlated with reported fatigue $\left(r_{p}=0.72 ; P=\right.$ 0.002; Fig. 1h). Since muscle relaxation is dependent upon the activity of sarco/endoplasmic reticulum $\mathrm{Ca}^{2+}$-ATPase (SERCA), an enzyme that mediates the re-uptake of calcium into the sarcoplasmic reticulum (SR) of skeletal muscle, SERCA activity was measured in whole-muscle homogenates [11]. However, no significant differences in SERCA activity were observed between CML + MC and CML - MC (98.9 (IQR 79.3-110.7 mU/mg) and 101.5 (IQR $77.0-109.8 \mathrm{mU} / \mathrm{mg}$ ), respectively; $P=0.97$ ).

To measure whole-body fitness levels, all subjects performed an incremental cycle ergometer test (Lode Excalibur; Groningen, the Netherlands) with continuous ECG monitoring [10] to assess peak oxygen uptake $\left(\mathrm{VO}_{2 \text { peak }}\right)$. $\mathrm{VO}_{2 \text { peak }}$ was $34.6 \pm 8.4 \mathrm{ml} / \mathrm{kg} / \mathrm{min}(100 \pm 9 \%$ predicted) in the CML patients, which was not different from the controls $\left(\mathrm{VO}_{2 \text { peak }} 35.6 \pm 7.5 \mathrm{ml} / \mathrm{kg} / \mathrm{min}, 100 \pm 9 \%\right.$ predicted; $P=$ $0.75)$. In addition, no differences in $\mathrm{VO}_{2 \text { peak }}$ were observed between CML + MC and CML - MC $(P=0.38)$.

Collectively, CML patients on TKI therapy show no signs of skeletal muscle mitochondrial dysfunction. However, quadriceps femoris muscle of TKI users fatigues to a larger extent upon repetitive stimulation when compared to controls. Changes in muscle contractile properties are associated with TKI-induced muscle complaints, as CML $+\mathrm{MC}$ show a significant lower maximal force rise and a tendency toward a delayed muscle relaxation after two minutes of electrical quadriceps femoris stimulations. CML patients did not have impaired maximal exercise performance.

On a cellular level, no effects of TKI therapy on skeletal muscle mitochondrial density and function were found. These results are in line with the only previous clinical case report in which two CML patients, who had to interrupt or reduce therapy with nilotinib because of muscle pain, failed to show disturbances in mitochondrial oxidative enzyme reactions [12]. Intriguingly, in vitro studies in $\mathrm{C} 2 \mathrm{C} 12$ myotubes showed no decline in ATP levels upon short-term imatinib incubation of $30 \mathrm{~min}$ [13], whereas long-term TKI-incubation of $24 \mathrm{~h}$ showed decreased ATP levels overtime [5, 13].

Disturbances in heart mitochondrial function are suggested to occur secondary to activation of a stress response in the endoplasmic reticulum [4]. Perhaps, in skeletal muscle, changes in the function of other cellular organelles also precede mitochondrial disturbances. In support of this hypothesis, CML patients on TKI therapy showed significantly more muscle fatigue than controls, and CML +
MC showed delayed quadriceps femoris muscle force generation and a trend toward delayed relaxation in fatigued muscle compared to CML - MC. Since muscle fatigability, force generation, and relaxation are largely dependent on $\mathrm{Ca}^{2+}$ regelulation by the SR, changes in SR functioning may underlie these findings [14]. In that respect, disturbances in $\mathrm{Ca}^{2+}$ homeostasis [15], and SR abnormalities (i.e., dilated SR with membrane whorls) [4] have been found upon imatinib treatment in myocytes, but have never been linked to muscle complaints. Although we found no difference in SERCA activity between CML + MC and CML - MC, muscle half relaxation time after 2-min stimulation correlated positively with the perception of fatigue in CML patients, and may therefore be an important key for understanding the mechanism underlying fatigue in CML.

To the best of our knowledge, maximal exercise capacity has not been assessed before in CML patients or other TKIusers. Compared to controls, CML patients do not have diminished maximal exercise capacity as measured by $\mathrm{VO}_{2 \text { peak }}$ and have similar physical activity levels as controls. $\mathrm{VO}_{2 \text { peak }}$ was also not different between $\mathrm{CML}+\mathrm{MC}$ and CML - MC, despite higher subjective fatigue levels in CML + MC. These findings fit with the unaltered mitochondrial ATP production capacity, which is an important determinant of $\mathrm{VO}_{2 \text { peak }}$.

There are several limitations to this study. Due to the exploratory character of the study a relatively large number of measurements were performed in a small sample size. Therefore, results should be cautiously interpreted. Nonetheless, this design made it possible to examine the influence of TKIs on multiple levels (i.e. cellular, muscle tissue and whole body level) which offers broad insight into the effects of TKIs in CML patients. Secondly, participants were only included when they were able to perform all study measurements. Thus patients who were unable to perform exercise testing were excluded. Consequently, extreme cases of TKIinduced skeletal muscle complaints were not included in this study, which may have underestimated the results.

This study provides important information concerning the effects of TKIs on skeletal muscle function and whole body fitness and lays foundation for further studies to elucidate the precise mechanism by which TKI therapy causes muscle complaints and affects muscle function.

Acknowledgements We would like to thank Berendien StoltenborgHogenkamp, Karina Horsting-Wethly and Theo van Lith of the Translational Metabolic Laboratory at the Radboudumc for their assistance with the mitochondrial measurements.

\section{Compliance with ethical standards}

Conflict of interest The authors declare that they have no conflict of interest. 
Publisher's note: Springer Nature remains neutral with regard to jurisdictional claims in published maps and institutional affiliations.

Open Access This article is licensed under a Creative Commons Attribution 4.0 International License, which permits use, sharing, adaptation, distribution and reproduction in any medium or format, as long as you give appropriate credit to the original author(s) and the source, provide a link to the Creative Commons license, and indicate if changes were made. The images or other third party material in this article are included in the article's Creative Commons license, unless indicated otherwise in a credit line to the material. If material is not included in the article's Creative Commons license and your intended use is not permitted by statutory regulation or exceeds the permitted use, you will need to obtain permission directly from the copyright holder. To view a copy of this license, visit http://creativecommons. org/licenses/by/4.0/.

\section{References}

1. Kekäle M, Peltoniemi M, Airaksinen M. Patient-reported adverse drug reactions and their influence on adherence and quality of life of chronic myeloid leukemia patients on per oral tyrosine kinase inhibitor treatment. Patient Prefer Adherence. 2015;9:1733-40.

2. Marin D, Bazeos A, Mahon F-X, Eliasson L, Milojkovic D, Bua $\mathrm{M}$, et al. Adherence is the critical factor for achieving molecular responses in patients with chronic myeloid leukemia who achieve complete cytogenetic responses on imatinib. J Clin Oncol. 2010;28:2381-8

3. Efficace F, Baccarani M, Breccia M, Cottone F, Alimena G, Deliliers GL, et al. Chronic fatigue is the most important factor limiting health-related quality of life of chronic myeloid leukemia patients treated with imatinib. Leukemia. 2013;27: 1511-9.

4. Kerkela R, Grazette L, Yacobi R, Iliescu C, Patten R, Beahm C, et al. Cardiotoxicity of the cancer therapeutic agent imatinib mesylate. Nat Med. 2006;12:908-16.

5. Will Y, Dykens JA, Nadanaciva S, Hirakawa B, Jamieson J, Marroquin LD, et al. Effect of the multitargeted tyrosine kinase inhibitors imatinib, dasatinib, sunitinib, and sorafenib on mitochondrial function in isolated rat heart mitochondria and H9c2 cells. Toxicol Sci. 2008;106:153-61.

6. Mendoza TR, Wang XS, Cleeland CS, Morrissey M, Johnson BA, Wendt JK, et al. The rapid assessment of fatigue severity in cancer patients: use of the Brief Fatigue Inventory. Cancer. 1999;85: 1186-96.

7. Wendel-Vos GC, Schuit AJ, Saris WH, Kromhout D. Reproducibility and relative validity of the short questionnaire to assess health-enhancing physical activity. J Clin Epidemiol. 2003;56: 1163-9.

8. Gibbons RJ, Balady GJ, Bricker JT, Chaitman BR, Fletcher GF, Froelicher VF, et al. ACC/AHA 2002 guideline update for exercise testing: summary article. A report of the American College of Cardiology/American Heart Association Task Force on Practice Guidelines (Committee to Update the 1997 Exercise Testing Guidelines). J Am Coll Cardiol. 2002;40:1531-40.

9. Charlson ME, Pompei P, Ales KL, MacKenzie CR. A new method of classifying prognostic comorbidity in longitudinal studies: development and validation. J Chronic Dis. 1987;40:373-83.

10. Allard NAE, Schirris TJJ, Verheggen RJ, Russel FGM, Rodenburg RJ, Smeitink JAM, et al. Statins affect skeletal muscle performance: evidence for disturbances in energy metabolism. J Clin Endocrinol Metab. 2018;103:75-84.

11. Gerrits HL, De Haan A, Hopman MT, van Der Woude LH, Jones DA, Sargeant AJ. Contractile properties of the quadriceps muscle in individuals with spinal cord injury. Muscle Nerve. 1999; 22:1249-56.

12. Caocci G, Maioli MA, Atzeni S, Piras R, Carboni N, La Nasa G. Absence of histological myopathy in chronic myeloid leukemia patients complaining of muscle spasms and myalgia during treatment with nilotinib. Leuk Res. 2012;36:e206-8.

13. Damaraju VL, Kuzma M, Cass CE, Putman CT, Sawyer MB. Multitargeted kinase inhibitors imatinib, sorafenib and sunitinib perturb energy metabolism and cause cytotoxicity to cultured C2C12 skeletal muscle derived myotubes. Biochem Pharmacol. 2018;155:162-71.

14. Westerblad H, Allen DG. Changes of myoplasmic calcium concentration during fatigue in single mouse muscle fibers. J Gen Physiol. 1991;98:615-35.

15. Barr LA, Makarewich CA, Berretta RM, Gao H, Troupes CD, Woitek $\mathrm{F}$, et al. Imatinib activates pathological hypertrophy by altering myocyte calcium regulation. Clin Transl Sci. 2014;7:360-7. 